\title{
Classificação de Blocos de Imagens de Padrões Radiológicos Pulmonares com Resampling SMOTE
}

\author{
Johanna Elisabeth Rogalsky ${ }^{1}$, Jeovane Honório Alves ${ }^{1}$, Lucas Ferrari de Oliveira ${ }^{1}$ \\ ${ }^{1}$ Universidade Federal do Paraná (UFPR) \\ Laboratório de Visão, Robótica e Imagem (VRI) \\ Curitiba - PR, Brasil \\ \{jer14, jhalves, lferrari\}@inf.ufpr.br
}

\begin{abstract}
Diffuse Pulmonary Diseases can affect the lung parenchyma, causing respiratory deficiencies and even cause almost complete loss of function, requiring a more accurate evaluation for a concrete diagnosis. Using computational techniques, the purpose of this work is to use feature descriptors (LBP, CLBP, gray-level histogram and GLCM) for classification of lung patterns, assisting radiologists in the diagnosis of these diseases. Using a patch-based approach, SMOTE resampling and the SVM classifier, accuracy of $87.41 \%$ and sensitivity of $88.31 \%$ were achieved.
\end{abstract}

Resumo. Doenças Pulmonares Difusas podem afetar o parênquima pulmonar, causando deficiências respiratórias até a quase completa perda de função, sendo necessária uma avaliação mais precisa para um diagnóstico concreto. Utilizando-se de técnicas computacionais, a proposta deste trabalho é utilizar descritores de características (LBP, CLBP, histograma de níveis de cinza e GLCM) para a classificação de padrões pulmonares, auxiliando radiologistas no diagnóstico dessas doenças. Utilizando uma abordagem baseada em blocos, resampling SMOTE e o classificador SVM, uma taxa de acerto de $87,41 \%$ e sensibilidade de $88,31 \%$ foram alcançadas.

\section{Introdução}

Dentre as diversas doenças que afetam os pulmões, estão as Doenças Pulmonares Difusas (DPDs). Elas afetam o parênquima pulmonar de forma que gradualmente causam deficiências pulmonares e somam mais de 150 diferentes diagnósticos histológicos [Depeursinge et al. 2012]. Além disso, devido a diferenças individuais dos pacientes, os padrões radiológicos que representam uma mesma DPD frequentemente apresentam dessemelhanças nos padrões nas imagens [Li et al. 2013].

Para obter uma avaliação mais precisa de padrões radiológicos, a Tomografia Computadorizada de Alta Resolução (TCAR) [Zhao et al. 2013] vem sendo aceita como o padrão-ouro para fins de diagnóstico [Alemzadeh et al. 2015]. Ademais, imagens de TCAR conseguem exibir combinações de padrões teciduais distintas para diferentes DPDs [Song et al. 2013], trazendo informações detalhadas que permitem uma melhor visualização do tecido [Kale et al. 2016].

No entanto, o grande número de cortes que as TCARs produzem torna a interpretação das imagens trabalhosa e complexa para médicos e radiologistas, sendo ela dependente da experiência dos mesmos [Zhao et al. 2013]. Dessa forma, sistemas de auxílio 
ao diagnóstico (CAD, do inglês Computer Aided Diagnosis) se tornam ferramentas importantes nesse processo. Pois além de poder complementar o diagnóstico ao identificar especificidades não evidentes a olho nu, essas ferramentas podem ajudar na detecção precoce de câncer [Firmino et al. 2014]. Por isso, os sistemas CADs vem tendo cada vez mais importância, uma vez que diagnósticos de exames por imagem trazem várias dificuldades na decisão para médicos e radiologistas.

\section{Trabalhos Relacionados}

[Xu et al. 2013] propuseram classificar seis padrões pulmonares (consolidação, vidrofosco, favo-de-mel, nodular, enfisema e tecido normal) em imagens de TCAR utilizando Bag-of-features e SVM. Essa abordagem atingiu uma de acurácia de 93,18\% e taxas de sensibilidade e precisão acima dos 90\%. Em [Zhao et al. 2015], um método de representação esparso das características locais extraídas de VOIs (Volume of interest) das imagens foi desenvolvido, de forma a codificar a informação semântica presente nelas. Utilizando o K-Means em conjunto com uma versão própria do OMP (Orthogonal Matching Pursuit) para cálculo de coeficientes do dicionário e cálculo da codificação esparsa com os mesmos, respectivamente. Esse método atingiu uma acurácia de 96,4\% para os mesmos padrões pulmonares do trabalho anterior.

Em [Song et al. 2015], é mostrada a abordagem de classificação Large Margin Local Estimate (LMLE), que ao incorporar um classificador de representação esparsa a uma construção de sub-categorização, consegue abordar de forma efetiva a variação intraclasse e a ambiguidade inter-classe. As regiões de interesse (ROI, do inglês Region of interest) analisadas são de imagens CT (Computed Tomography) de padrões radiológicos com tecidos pulmonares (normal, enfisema, vidro-fosco, fibrose e micro-nódulos), representadas por um vetor de texture-intensity-gradient. Esse classificador (LMLE) atingiu uma acurácia de $90 \%$. O trabalho de [ONO et al. 2017] compara métodos convencionais, os métodos de modelagem esparsos e o método baseado em Markov-Chain Monte Carlo (MCMC) para seleção de características em termos de classificação, mostrando uma acurácia média de $92 \%$.

Apesar de diversos trabalhos apresentarem resultados interessantes, é necessário ainda aprimorá-los para que possam ser utilizados no ambiente clínico. O presente trabalho tem por objetivo utilizar descritores de características de textura e baseados em histograma(s) aplicados em blocos de imagens, visando classificar corretamente com abordagens simples e diretas. Além disso, aplicar as técnicas para balanceamento de amostras (resampling). A Seção 3 apresenta a base estudada, assim como os métodos aplicados. Resultados preliminares são apresentados na Seção 4. Por fim, uma conclusão inicial e trabalhos futuros são mostrados na Seção 5.

\section{Materiais e Métodos}

\subsection{Base de imagens}

A base de dados utilizada neste trabalho foi criada e disponibilizada por [Depeursinge et al. 2012]. Ela é composta de 113 exames com casos de DPDs dos Hospitais Universitários de Genebra, variando entre 11 a 61 cortes com resolução de 512 x 512 pixels. Além disso, a base provê ROIs demarcadas por radiologistas, referentes a 17 padrões pulmonares. 
A partir dela, uma nova base foi proposta por [Bagesteiro 2015], contendo blocos de imagens com tamanhos menores para serem analisados, visando uma análise mais local. A extração desses blocos foi feita a partir das ROIs delimitadas, com $75 \%$ dos pixels pertencentes a um mesmo padrão. Totalizando 20540 blocos rotulados em 6 classes (não-pulmão - NP, normal - N, enfisema - E, vidro-fosco - VF, fibrose - F e micro-nódulos - MN), das quais apenas cinco serão utilizadas, sendo a classe NP removida.

Desse modo, é possível verificar a classificação apenas de padrões que contenham tecido pulmonar, sendo o tamanho dos blocos de 32 x 32 pixels com a melhor troca entre o desempenho de classificação e localização. Além disso, as imagens de blocos estão divididas em 90 conjuntos, dos quais cada um representa um paciente. Na Figura 1 podem ser vistos exemplos de blocos de imagens com padrões pulmonares representando cada classe.

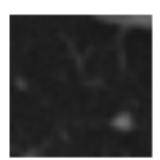

(a)

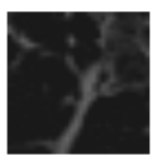

(b)

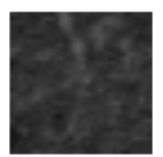

(c)

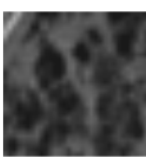

(d)

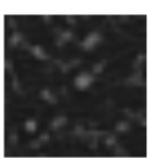

(e)

Figura 1. Exemplo dos blocos (a) pulmão normal, (b) enfisema, (c) vidro-fosco, (d) fibrose e (e) micro-nódulos.

\subsection{Abordagem proposta}

Um critério importante para que o sistema CAD possa classificar as imagens de forma satisfatória é a extração de características que representem eficientemente as estruturas e características das ROIs utilizadas. Neste trabalho, os descritores LBP (Local Binary Pattern), CLBP (Completed Local Binary Pattern), Matriz de Co-ocorrência de Níveis de Cinza (GLCM, do inglês Gray Level Co-occurrence Matrix), histograma e estatísticas de histograma foram utilizados [Guo et al. 2010, Haralick et al. 1973, Gonzalez and Woods 2002].

Como as amostras da base estão desbalanceadas, foram aplicadas as técnicas de SMOTE [Chawla et al. 2002], para aumentar o número de amostras para as classes com poucos exemplos, e undersampling aleatório, para diminuir o número de amostras para as que apresentam exemplos em excesso. A comparação da quantidade de amostras por classe pode ser vista na Tabela 1.

\begin{tabular}{l||cc}
\multicolumn{1}{c||}{ Padrão } & $\begin{array}{c}\text { Quantidade } \\
\text { original }\end{array}$ & $\begin{array}{c}\text { Quantidade } \\
\text { utilizada }\end{array}$ \\
\hline \hline Normal & 5733 & 4000 \\
\hline Enfisema & 1017 & 3500 \\
\hline Vidro-fosco & 1942 & 3500 \\
\hline Fibrose & 2736 & 3500 \\
\hline Micronódulos & 6112 & 4000
\end{tabular}

Tabela 1. Quantidade original de vetores e quantidade obtida após o balanceamento. 
O classificador de Máquina de Vetores de Suporte [Cortes and Vapnik 1995] (SVM, do inglês Support Vector Machine) foi utilizado para a classificação dos blocos. Para a otimização dos seus hiper-parâmetros, a técnica de busca aleatória (do inglês Random Search) foi utilizada, por obter bons valores com rapidez, se comparado com técnicas como Grid-Search [Bergstra and Bengio 2012]. Para a avaliação da abordagem proposta, a técnica de leave-one-patient-out foi aplicada (para cada paciente da base, suas amostras são retiradas para avaliação e as amostras de outros pacientes são usadas no treinamento).

\section{Resultados e Discussão}

Para a avaliação dos descritores, as métricas de sensibilidade, precisão, especificidade e acurácia foram utilizadas. Os resultados de cada descritor podem ser vistos na Tabela 2, ordenadas em ordem decrescente de sensibilidade.

\begin{tabular}{l||cccc}
\hline Classificador & Sensibilidade & Precisão & Especificidade & Acurácia \\
\hline \hline CLBP & 88,31 & 88,53 & 96,70 & 87,41 \\
\hline Histograma & 75,92 & 75,97 & 92,53 & 76,09 \\
\hline LBP & 70,15 & 69,37 & 90,33 & 76,72 \\
\hline GLCM & 63,55 & 64,91 & 87,36 & 64,31 \\
\hline Medidas estatísticas & 45,53 & 40,70 & 80,16 & 50,51 \\
\hline
\end{tabular}

Tabela 2. Resultados gerais dos classificadores (em \%).

É possível verificar que o melhor descritor nos experimentos executados foi o CLBP. Ele representa a textura em três componentes e combina o mapa das três em uma única componente final, o histograma. Descrevendo assim, a textura de forma mais completa e portanto, melhora a acurácia significativamente. Além disso, a diferença de sensibilidade para o próximo descritor é de $12,39 \%$. O próximo extrator a conseguir uma boa taxa de reconhecimento é o histograma. Ele descreve a imagem de acordo com a frequência de seus níveis de cinza, mostrando assim a distribuição da textura na imagem. Dessa forma, atinge melhores resultados que as suas medidas estatísticas, condizendo com o apresentado por [Depeursinge et al. 2012].

\begin{tabular}{l||c|c|c|c|c}
\hline \multirow{2}{*}{ Classe } & \multicolumn{6}{|c}{ Classe Predita } & & & \\
\cline { 2 - 6 } & $\mathrm{N}$ & $\mathrm{E}$ & $\mathrm{VF}$ & $\mathrm{F}$ & $\mathrm{MN}$ \\
\hline \hline $\mathrm{N}$ & 86,22 & 1,38 & 6,75 & 0,43 & 5,22 \\
\hline $\mathrm{E}$ & 2,11 & 96,77 & 0,37 & 0,71 & 0,03 \\
\hline $\mathrm{VF}$ & 9,63 & 0,23 & 85,06 & 3,89 & 1,20 \\
\hline $\mathrm{F}$ & 1,51 & 2,46 & 10,60 & 83,00 & 2,43 \\
\hline $\mathrm{MN}$ & 6,55 & 0,23 & 1,48 & 1,22 & 90,53
\end{tabular}

Tabela 3. Matriz de confusão dos momentos do CLBP (em \%).

A partir da Tabela 3, que mostra a matriz de confusão do CLBP, verifica-se que o descritor obtem melhores resultados para as classes enfisema e micro-nódulos, conseguindo taxas parecidas com trabalhos já realizados com a mesma base ([Xu et al. 2013], [Song et al. 2015]). Observa-se que o pior resultado é da classe fibrose, ocorrendo a maior confusão com vidro-fosco, o que, de acordo com vários trabalhos na literatura, é bastante 
comum por apresentarem padrões teciduais parecidos. Além disso, sua acurácia é de $87,41 \%$, apesar de sua taxa de especificidade de $96,70 \%$. Ademais, a combinação de suas três componentes traz a precisão de $88,53 \%$, com sensibilidade de 88,31\%.

Para o histograma, a melhor taxa também foi da classe enfisema e ao contrário do CLBP, fibrose não é a classe com a pior taxa de acerto, mas a segunda melhor, sendo a classe com pior desempenho a classe normal por conta do seu histograma, que possui menos variações nos níveis de cinza. Os resultados obtidos foram mais altos e diferentes do que o esperado se comparado com o trabalho de [Bagesteiro 2015], possivelmente pelo balanceamento da base de amostras. Além disso, obteve taxa de especificidade de $92,53 \%$.

O LBP também obteve melhor taxa de acerto com as classes enfisema e micronódulos, onde as maiores confusões dessas classes ocorrem com o padrão normal. Além disso, sua pior taxa ocorre na classe normal, também por não apresentar muita textura. Teve uma precisão de $69,37 \%$ e sensibilidade de $70,15 \%$, que são resultados melhores do que os encontrados em [Bagesteiro 2015], mostrando que tanto no histograma, como no LBP, o balanceamento da base melhorou os resultados.

\section{Conclusão e Trabalhos Futuros}

Diagnósticos precisos de DPDs através de imagens de TC ou TCAR são muito importantes, porém muito trabalhosos e cansativos para médicos e radiologistas. Para isso, vem-se desenvolvendo ferramentas para auxiliar nesse processo, os sistemas CADs. Dessa forma, o presente trabalho propôs métodos de extração de características dessas imagens no intuito, de reconhecer as doenças presentes e então classificá-las.

Sendo assim, a contribuição desse trabalho é mostrar, no ambiente proposto pelo mesmo, que o extrator CLBP alcançou as melhores taxas. Cujos valores de sensibilidade e especifidade são de $88,31 \%$ e de $96,70 \%$, respectivamente. Ademais, para próximas etapas, propõe-se realizar o mesmo processo, mas com uma fusão hierárquica de classificadores de acordo com a maior taxa de acerto de cada um. Além disso, analisar o resultado de outros descritores, assim como analisar as diferenças entre uma abordagem usando redes neurais convolucionais, que está sendo bem utilizada inclusive na área de images médicas.

\section{Referências}

Alemzadeh, M., Boylan, C., and Kamath, M. V. (2015). Review of texture quantification of ct images for classification of lung diseases. Critical Reviews ${ }^{T M}$ in Biomedical Engineering, 43(2-3).

Bagesteiro, L. D. (2015). Classificação de padrões radiológicos por blocos em imagens não segmentadas de tomografia computadorizada.

Bergstra, J. and Bengio, Y. (2012). Random search for hyper-parameter optimization. Journal of Machine Learning Research, 13(Feb):281-305.

Chawla, N. V., Bowyer, K. W., Hall, L. O., and Kegelmeyer, W. P. (2002). Smote: synthetic minority over-sampling technique. Journal of artificial intelligence research, $16: 321-357$. 
Cortes, C. and Vapnik, V. (1995). Support-vector networks. Machine learning, 20(3):273297.

Depeursinge, A., Vargas, A., Platon, A., Geissbuhler, A., Poletti, P.-A., and Müller, H. (2012). Building a reference multimedia database for interstitial lung diseases. Computerized medical imaging and graphics, 36(3):227-238.

Firmino, M., Morais, A. H., Mendoça, R. M., Dantas, M. R., Hekis, H. R., and Valentim, R. (2014). Computer-aided detection system for lung cancer in computed tomography scans: review and future prospects. Biomedical engineering online, 13(1):41.

Gonzalez, R. C. and Woods, R. E. (2002). Digital Image Processing. Addison-Wesley Longman Publishing Co., Inc., Boston, MA, USA, 2nd edition.

Guo, Z., Zhang, L., and Zhang, D. (2010). A completed modeling of local binary pattern operator for texture classification. IEEE Transactions on Image Processing, 19(6):1657-1663.

Haralick, R. M., Shanmugam, K., et al. (1973). Textural features for image classification. IEEE Transactions on systems, man, and cybernetics, SMC-3(6):610-621.

Kale, M., Mukhopadhyay, S., Dash, J. K., Garg, M., and Khandelwal, N. (2016). Differentiation of several interstitial lung disease patterns in hrct images using support vector machine: role of databases on performance. In Medical Imaging 2016: ComputerAided Diagnosis, volume 9785, page 97852Z. International Society for Optics and Photonics.

Li, Q., Cai, W., and Feng, D. D. (2013). Lung image patch classification with automatic feature learning. In Engineering in Medicine and Biology Society (EMBC), 2013 35th Annual International Conference of the IEEE, pages 6079-6082. IEEE.

ONO, S., KOIWAI, M., SHOUNO, H., and KIDO, S. (2017). Comparison of feature selection method for diffuse lung disease. PDPTA.

Song, Y., Cai, W., Huang, H., Zhou, Y., Feng, D. D., Wang, Y., Fulham, M. J., and Chen, M. (2015). Large margin local estimate with applications to medical image classification. IEEE transactions on medical imaging, 34(6):1362-1377.

Song, Y., Cai, W., Zhou, Y., and Feng, D. D. (2013). Feature-based image patch approximation for lung tissue classification. IEEE transactions on medical imaging, 32(4):797-808.

Xu, R., Hirano, Y., Tachibana, R., and Kido, S. (2013). A bag-of-features approach to classify six types of pulmonary textures on high-resolution computed tomography. IEICE TRANSACTIONS on Information and Systems, 96(4):845-855.

Zhao, W., Xu, R., Hirano, Y., Tachibana, R., and Kido, S. (2013). Classification of diffuse lung diseases patterns by a sparse representation based method on hrct images. In Engineering in Medicine and Biology Society $(E M B C), 2013$ 35th Annual International Conference of the IEEE, pages 5457-5460. IEEE.

Zhao, W., Xu, R., Hirano, Y., Tachibana, R., and Kido, S. (2015). A sparse representation based method to classify pulmonary patterns of diffuse lung diseases. Computational and mathematical methods in medicine, 2015. 